\title{
QUANTUM INTERFEROMETRY, MEASUREMENT AND OBJEC- TIVITY: SOME BASIC FEATURES REVISITED
}

\author{
Markus Simonius \\ Institut für Teilchenphysik, Eidgenössische Technische Hochschule, \\ CH-8093 Zürich, Switzerland
}

The reduction paradigm of quantum interferometry and the objectivation problem in quantum measurements are reanalyzed. Both are shown to be amenable to straightforward mathematical treatment within "every-users" simple-minded quantum mechanics without reduction postulate etc., using only its probabilistic content.

Key words: quantum interferometry, state reduction, measurement, objective events.

\section{INTRODUCTION: POSTULATES AND INTERPRETATION}

Everyusers quantum mechanics, based only on it's minimal probabilistic interpretation, is a concise and extremely successful theory without any intrinsic contradictions. Difficulties usually arise only when additional interpretational assumptions are introduced in order to bridge missing links in its understanding.

It is the object of this paper to demonstrate that once the basic axioms expressing the probabilistic content of quantum theory are laid down problems can be solved by careful definitions and simple but rigorous mathematics, without invoking any additional ad hoc ingredients.

Only the minimal probabilistic postulates I and II of quantum mechanics listed below will be used. No state reduction and no axiom of measurement etc.! Postulate III is included for completeness. It is not used but not violated either.

$I$ States of a physical system are represented by elements $X$ of the convex set $\mathcal{S}$ of density matrices on a Hilbert space $\mathcal{H}$ characterized by $X \geq 0$ and $\operatorname{Tr}(X)=1$.

II The probability for any given kind of event for a system in a state represented by $X$ is expressed by an expectation value $(A, X) \equiv \operatorname{Tr}[A X]$ where $A$ is a hermitian operator on $\mathcal{H}$, called an observable, which obviously must obey $0 \leq A \leq 1$ or, equivalently, $0 \leq\langle\varphi|A| \varphi\rangle \leq 1$ for all $\varphi \in \mathcal{H},\|\varphi\|=1$. The set of all such operators is denoted by $\widehat{\mathcal{O}}$.

III The evolution of an isolated system is reversible on $\mathcal{S}$ and thus unitary on $\mathcal{H}$.

Note the important fact that $(A, X)$ is convex linear on $\mathcal{S}$ under mixing, $\left(A,\left|c_{1}\right|^{2} X_{1}+\right.$ $\left.\left|c_{2}\right|^{2} X_{2}\right)=\left|c_{1}\right|^{2}\left(A, X_{1}\right)+\left|c_{2}\right|^{2}\left(A, X_{2}\right)$, as required by its interpretation as a probability and that any such function can be represented by some $A \in \widehat{\mathcal{O}}$.

\footnotetext{
${ }^{1}$ Presented at the 2nd INTERNATIONAL SYMPOSIUM ON FUNDAMENTAL PROBLEMS IN QUANTUM PHYSICS, Oviedo, Spain, 21-26 July 1996 (Kluwer Academic Publ., in press).
} 
For a pure state $X=|\varphi\rangle\langle\varphi|$ with $\varphi \in \mathcal{H},\|\varphi\|=1$ and $(A, X)=\langle\varphi|A| \varphi\rangle$. Pure states play no conceptually distinguished role, and in particular it is not assumed that a system is always in some, perhaps unknown, pure state.

In II above "probability" is just a number between zero and one expressible by a formula within the theory. This number is interpreted as the probability for the occurrence of something named an event $e \in\{1,0\}$ where $e=1$ means that the event occurs in a given trial and $e=0$ that it does not. There is no corresponding formula for events, however, and actually none is needed for any practical application. Nevertheless, it will be shown that events can be described in quantum mechanics using only I and II above and without violating III, where an event is defined by the following objectivity criterion [1]:

The basic property defining an event and guaranteeing its objectivity is that it can be observed or read in at least two independent, mutually non-interfering ways with necessarily agreeing results (zero probability for disagreement).

\section{SINGLE QUANTUM INTERFEROMETRY [2]}

Consider a typical interferometer arrangement as sketched in Fig. 1. Its properties are well known and often discussed: even if only one quantum (photon or neutron etc.) is inside the arrangement at a given time, the configuration of Fig. 1a can reveal interference between the states passing the two arms I and II provided none of them is blocked. Correspondingly, a pure single-particle state within the interferometer is represented by a normed wave function of the form

$$
\varphi=c_{1} \varphi_{1}+c_{2} \varphi_{2}, \quad\|\varphi\|=\left\|\varphi_{1}\right\|=\left\|\varphi_{2}\right\|=1
$$

where, $\varphi_{1}$ and $\varphi_{2}$ represent states passing completely along one of the two paths I or II, respectively, in the interferometer and have zero component in the other (and thus are mutually orthogonal). For ideal 50:50 beam-splitting $\left|c_{1}\right|^{2}=\left|c_{2}\right|^{2}=\frac{1}{2}$.

Now consider instead coincidences between two detectors inserted into the two paths of the interferometer as shown in Fig. 1 $1 \mathrm{~b}$. From the appearance of the wave function (11) one might suspect that such coincidences should occur. But quanta (particles or photons) are supposed to be indivisible entities and thus a single quantum is not able to trigger both detectors in the arrangement of Fig. 1 $1 \mathrm{~b}$ (nor in Fig. 17a, of course). Though all this is much discussed textbook wisdom, one looks in vain in the literature for a satisfying derivation of this fact which uses only the basic structure of single particle quantum theory and does not amount to just stating the fact as postulate in one way or another. But clearly it should be

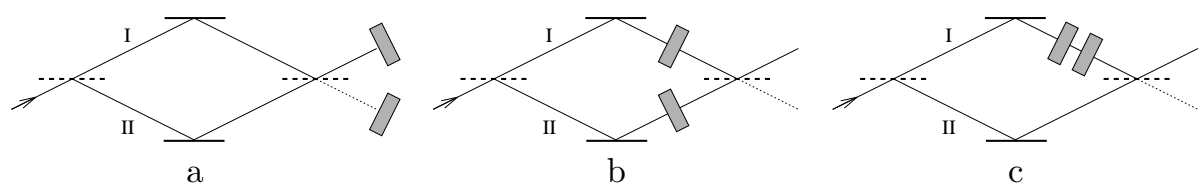

Figure 1: Sketch of typical interferometer arrangements with differently placed detectors. 
possible to deduce such basic properties mathematically once the general premises of the theory are laid down [3]. And this, in fact, turns out to be the case.

The analysis is based on the following "silly" theorem which is a simple consequence of the positivity postulated in II:

Theorem 1: Let $A$ be a positive operator on $\mathcal{H}$ such that $\left\langle\psi_{1}|A| \psi_{1}\right\rangle=\left\langle\psi_{2}|A| \psi_{2}\right\rangle=$ 0 for given $\psi_{1}, \psi_{2} \in \mathcal{H}$. Then $\langle\psi|A| \psi\rangle=0$ for all superpositions $\psi=c_{1} \psi_{1}+c_{2} \psi_{2}$ between them.

In order to apply this theorem to Fig. 1 $1 \mathrm{~b}$ with the two detectors in coincidence one only has to remark that one of the fundamental requirements for a coincidence between the two detectors is that the probability for a coincidence event be zero for any state which has zero component in one of the two arms of the interferometer, i.e if either $c_{1}=0$ or $c_{2}=0$ in Eq. (11). (This is actually what careful experimenters check in order to verify that their arrangement does not produce spurious coincidence events!) Thus $\left\langle\varphi_{1}|A| \varphi_{1}\right\rangle=\left\langle\varphi_{2}|A| \varphi_{2}\right\rangle=0$ where $A$ is the operator describing the probability of coincidence events. It then follows from the above theorem that $\langle\varphi|A| \varphi\rangle=0$ also for arbitrary $\varphi=c_{1} \varphi_{1}+c_{2} \varphi_{2}$ and thus that also for arbitrary superpositions between the states in the two arms of the interferometer the two

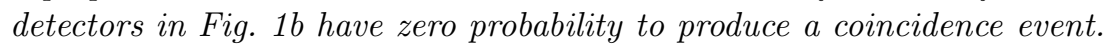

A corresponding "reduction theorem" is obtained similarly (using anticoincidence between the two detectors, this time) for an arrangement of the kind shown in Fig. 1c where now both detectors are in the same path and it is assumed that at least the first detector transmits (does not absorb) the quanta: If the two detectors have unit efficiency either both of them fire or none. Of course two-slit or Stern-Gerlach arrangements can be analyzed correspondingly.

Thus "what must be" is graciously born out by the mathematical analysis in spite of appearance of the wave function in Eq. (1), "with nothing left to the discretion of the theoretical physicist" [3] (except to formulate the problems properly). Astonishingly, in spite of its simplicity, no such treatment is found in the literature, let alone in textbooks where it would belong.

The structure of theorem 1 and of the corresponding physical statements is emphasized: Conclusions for arbitrary superpositions between two states $\varphi_{i}$ are obtained from conditions imposed only for the two states $\varphi_{i}$ themselves. There is no need to postulate what happens in the case of superpositions.

\section{MEASUREMENTS AND OBJECTIVE EVENTS [1]}

The demonstration of the emergence of objective events is conceptually more involved and only a rather condensed exposition of the concepts and main result can be given here. For a more general and detailed discussion refer to Ref. [1].

The following operational definitions of discrimination and superpositions [1], formulated for density operators and probabilities directly, should be visualized with the help of the different arrangements of Fig. 1. and related to the more familiar formulations in terms of Hilbert space elements in case of pure states.

An observable $A \in \widehat{\mathcal{O}}$ discriminates between two states $X_{1}, X_{2}$ if $\left(A, X_{1}\right)=1$ and $\left(A, X_{2}\right)=0$ or vice versa. Such $A$ exists provided $X_{1}$ and $X_{2}$ are orthogonal.

A state $X$ is a (general) superposition between two orthogonal states $X_{1}$ and 
$X_{2}$ with some fixed weights $\left|c_{1}\right|^{2}$ and $\left|c_{2}\right|^{2}$ such that $\left|c_{1}\right|^{2}+\left|c_{2}\right|^{2}=1$ if

$$
\begin{array}{ll}
(A, X)=\left|c_{2}\right|^{2}\left(A, X_{2}\right) & \forall A \in \widehat{\mathcal{O}}:\left(A, X_{1}\right)=0 \\
(A, X)=\left|c_{1}\right|^{2}\left(A, X_{1}\right) & \forall A \in \widehat{\mathcal{O}}:\left(A, X_{2}\right)=0
\end{array}
$$

The set of all states $X$ with this property is denoted by $\mathcal{S}\left(\left|c_{1}\right|^{2} X_{1},\left|c_{2}\right|^{2} X_{2}\right)$. It is obviously convex and contains the incoherent mixture $\left|c_{1}\right|^{2} X_{1}+\left|c_{2}\right|^{2} X_{2}$.

An observable $A$ is sensitive to interference between $X_{1}$ and $X_{2}$ if $(A, X) \neq$ $\left(A,\left|c_{1}\right|^{2} X_{1}+\left|c_{2}\right|^{2} X_{2}\right)$ for some $X \in \mathcal{S}\left(\left|c_{1}\right|^{2} X_{1},\left|c_{2}\right|^{2} X_{2}\right)$.

An immediate consequence of this definition of superpositions is the following generalization of theorem 1 (and thus its implications!) to non-pure states:

Theorem 1': If in an arrangement $\left(A, X_{1}\right)=\left(A, X_{2}\right)=0$ for two orthogonal states $X_{1}, X_{2}$, then $(A, X)=0$ also for any superposition $X$ between these two states.

A central role plays the careful analysis and definition of the concept of measurement: In a measurement information on the state $X$ of an object is distributed onto different, at least two, separated channels (identified by greek upper indices) consisting of different systems on which mutually non-disturbing observations, using channel observables or readings $A^{\mu} \in \mathcal{O}^{\mu}, \mu=1,2, \ldots$, may be performed.

A given measurement is characterized by a function $m\left(\left\{A^{\mu}, \mu \in M\right\} ; X\right)$ : $\otimes_{\mu \in M} \mathcal{O}^{\mu} \otimes \mathcal{S} \rightarrow[0,1]$ where $M$ is the subset of channels which are read. It will be denoted also $m\left(A^{\mu} ; X\right)$ in case only one channel $\mu$ is read or $m\left(A^{\mu}, A^{\nu} ; X\right)$ in case two channels $\mu$ and $\nu$ are read simultaneously etc.. It is to be interpreted as the probability for coincidence events $\prod e^{\mu}, \mu \in M$, i.e $m\left(\left\{A^{\mu}, \mu \in M\right\} ; X\right)=$ $\operatorname{Prob}\left(\prod_{\mu \in M} e^{\mu}=1\right)$ where $e^{\mu}$ is the event obtained by the reading $A^{\mu}$.

The function $m$ must be (convex) linear in $\mathcal{S}$ and all $\mathcal{O}^{\mu}$ in analogy to the corresponding properties of $(A, X)$ appropriate for probabilities as discussed. In addition it has to fulfill the following important requirement expressing separability or mutual non-disturbance of readings of different channels:

$$
m\left(A^{\mu}, A^{\nu} ; X\right)+m\left(A^{\mu}, \bar{A}^{\nu} ; X\right)=m\left(A^{\mu} ; X\right)
$$

$(\mu \neq \nu)$ for all $A^{\mu, \nu} \in \mathcal{O}^{\mu, \nu}$ independent of $A^{\nu}$, and correspondingly for an arbitrary number of channels. Here the complement $\bar{A}$ of an observable $A$ is defined by $(\bar{A}, X)=1-(A, X)$ for arbitrary state $X$ which can be thought of as being the observable obtained from $A$ by the replacement of the corresponding event $e$ by its negation $\bar{e}=1-e$ (i. e. by inverting the output). The l.s.h of Eq. 3 then means that the event corresponding to $A^{\nu}$ is ignored though it has been obtained, and the r.h.s that no observation of channel $\nu$ is performed at all.

A channel $\mu$ of a measurement discriminates between $X_{1}$ and $X_{2}$ if reading of that channel alone, without further observation, allows one to discriminate between $X_{1}$ and $X_{2}$. A corresponding reading $A^{\mu}$ discriminates $X_{1}$ against $X_{2}$ if $m\left(A^{\mu} ; X_{1}\right)=1$ and $m\left(A^{\mu} ; X_{2}\right)=0$. This defines what a measurement is good for.

The fact that for arbitrary fixed readings $A^{\mu}, \mu \in M$ a measurement realizes some observable $A \in \widehat{\mathcal{O}}$ such that $(A, X)=m\left(\left\{A^{\mu}, \mu \in M\right\} ; X\right)$ together with the defining Eq. (2) of superpositions then leads to the central result:

Theorem 2 (Probability and Objectivity): Consider a measurement with readings $A^{\mu}, A^{\nu}$ for two different channels which both discriminate $X_{1}$ against $X_{2}$ and 
assume that $X \in \mathcal{S}\left(\left|c_{1}\right|^{2} X_{1},\left|c_{2}\right|^{2} X_{2}\right)$ is any superposition between the $X_{i}$. Then

$$
m\left(A^{\mu}, A^{\nu} ; X\right)=m\left(A^{\mu} ; X\right)=m\left(A^{\nu} ; X\right)=\left\|c_{1}\right\|^{2}
$$

and

$$
m\left(A^{\mu}, \overline{A^{\nu}} ; X\right)=m\left(\overline{A^{\mu}}, A^{\nu} ; X\right)=0
$$

implying that for all $X \in \mathcal{S}\left(\left|c_{1}\right|^{2} X_{1},\left|c_{2}\right|^{2} X_{2}\right)$ two such discriminating readings performed on separate channels have zero probability to disagree. The last statement is exactly the defining property of an objective event as given in section 1 which leads to the following

Corollary (Objective Events): A measurement which discriminates between two states $X_{1}$ and $X_{2}$ according to the condition of theorem 2, performed on any superposition $X$ between them, leads for each trial to an (objective) event for an observable $A$ which discriminates between $X_{1}$ and $X_{2}$.

The value of the event is not predicted in general, only its probability according to Eq. (4). However, under the condition of theorem 2, the probabilistic element is completely eliminated in the correlation between two discriminating channels. Reading one of them one can or could with certainty predict the outcome of an eventual reading of the other: "The moon is objectively there even if nobody looks."

The mutual independence of the readings of different channels required by the definition of objectivity is contained in the important separability condition (3). Besides this, the central ingredient of the proof of Eq. (5) is (again) theorem 1': The condition of theorem 2 together with the separability condition (3) which yields $m\left(A^{\mu}, \overline{A^{\nu}} ; X\right) \leq m\left(A^{\mu} ; X\right)$ etc. implies $m\left(A^{\mu}, \bar{A}^{\nu} ; X_{i}\right)=m\left(\overline{A^{\mu}}, A^{\nu} ; X_{i}\right)=0$ which then must hold for any superposition $X$ between $X_{1}$ and $X_{2}$ by theorem $1^{\prime}$.

Thus objective events emerge automatically from the definition of measurements. It is emphasized that only the mathematical properties of the functions $m(\cdot ; \cdot)$ and $(\cdot, \cdot)$ are actually used in the proof without reference to their interpretation in terms of events. Moreover, only the response of each channel separately to the two states $X_{i}$ is presupposed and, as in section 2, nothing about superpositions between them nor about correlations among different channels.

It can be shown [1] that the definition and emergence of objective events does not violate any principle of quantum mechanics. The rational behind this is quite simple: One just cannot violate the defining equations (A) of superpositions however complicated one chooses the arrangement for an observation to be.

The crucial feature for objectivity and consistency turns out to be the distribution of the information onto different channels. In the classical domain this happens continuously due to the abundance of channels consisting of light.

\section{REFERENCES}

1. M. Simonius, Helv. Phys. Acta 66, 721 (1993); 65, 884 (1992).

2. M. Simonius, Helv. Phys. Acta 69 Separanda, 15 (1996).

3. J. S. Bell, Physics World 3 No. 8, August 1990, p. 33. 